\title{
Erratum zu: Optimale Schlaganfallprävention bei geriatrischen Patienten mit Vorhofflimmern. Konsens- bericht eines interdisziplinären Expertengremiums
}

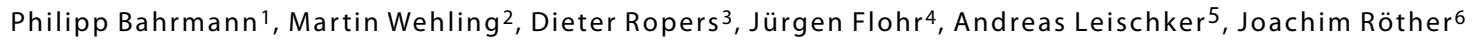 \\ 1 Institut für Biomedizin des Alterns der Friedrich-Alexander-Universität Erlangen-Nürnberg \\ 2 Institut für Experimentelle und Klinische Pharmakologie und Toxikologie, Medizinische Fakultät Mannheim, Ruprecht-Karls-Universität Heidelberg \\ ${ }^{3}$ Med. Klink I, Martha-Maria Krankenhaus, Nürnberg \\ ${ }^{4}$ Allgemeinmedizinische Gemeinschaftspraxis, Leipzig \\ ${ }^{5}$ Klinik für Allgemeine Innere Medizin und Altersmedizin, Alexianer Krefeld GmbH, Krefeld \\ ${ }^{6}$ Neurologische Abteilung, Asklepios Klinik Altona, Hamburg
}

MMW-Fortschr Med 2014 Oct 9; 156 Suppl3:84-88

http://dx.doi.org/10.1007/s15006-014-3480-1

Die Publikation erschien ohne den Hinweis, dass es sich dabei um eine Zusammenfassung handelt. Die englischsprachige, ausführliche Originalarbeit mit dem Titel „Optimal Stroke Prevention in the Geriatric Patient with Atrial Fibrillation: Position Paper of an Interdisciplinary Expert Panel“" ist in Drug Res (Stuttg). 2014 Oct 6 [Epub ahead of print] DOI: 10.1055/s-0034-1389984 erschienenen.

Wir bitten, den Fehler zu entschuldigen.

\author{
Korrespondenzadresse: \\ Priv.-Doz. Dr. med. Philipp Bahrmann, \\ MHBA, FESC \\ Institut für Biomedizin des Alterns \\ derFriedrich-Alexander-Universität \\ Erlangen-Nürnberg \\ Kobergerstraße 60 \\ 90408 Nürnberg \\ philipp.bahrmann@gmail.com
}

